\title{
Le Cursus Romand de Médecine de famille
}

\section{Sébastien Martin ${ }^{a}$ Bernard Giorgis ${ }^{b}$}

a Médecin de famille, médecin agréé, coordinateur du CRMF

b Médecin de famille, médecin agréé, coordinateur du CRMF

Correspondance:

Dr Sébastien Martin

Policlinique Médicale

Universitaire

Rue du Bugnon 44

CH-1011 Lausanne

Tél. 0213146116

sebastien.martin[at]hospvd.ch

\section{Actualité dans la formation postgraduée en médecine de famille}

En 2013, l'optimisme est de mise en matière de formation postgraduée en Suisse romande. On constate qu'un nombre croissant de médecins en formation s'oriente vers la médecine de famille. C'est le fruit des efforts entrepris depuis près de 10 ans par les associations de médecins de famille en collaboration avec les institutions concernées pour améliorer l'attrait du métier de médecin de famille auprès des médecins en formation, étudiants et assistants.

Ces futurs collègues ont besoin du soutien des médecins installés pour défendre une formation de qualité, centrée sur leurs besoins et des conditions de vie et de travail favorables en vue de leur installation. C'est aujourd'hui un des enjeux du masterplan pour la médecine de famille dont un des volets est le soutien à la formation postgraduée.

Depuis 5 ans, le Cursus Romand de Médecine de famille est le relai entre les médecins pratiquant la médecine de famille sur le terrain, leurs associations et les médecins en formation. A l'heure actuelle, près de 250 médecins en formation postgraduée dans toute la Suisse romande bénéficient du soutien et des conseils du CRMF.

Le $1^{\text {er }}$ février 2013, à l'heure où les associations faîtières ont unanimement adopté l'appellation «médecin de famille», le cursus romand fait peau neuve, change d'image et devient Cursus Romand de Médecine de Famille (voir figure ci-dessus).

\section{Tableau 1}

Les coordinateurs du programme sont rattachés soit aux Facultés de Médecine de Lausanne et Genève soit aux cantons et programmes intégrés dans toute la Suisse romande.

\section{Coordinateurs romands \\ Dr B. Giorgis \\ Dr S. Martin}

Coordinateurs cantonaux

BE: Dr F. Gerber

FR: Dr J. Zimmermann

JU: Dr J. Gainon

NE: Dr J. Rillot

VS: Dr P. Della Bianca

VD: Drs O. Pache et P. Raimondi

\section{Bureau}

Mme P. Rajaonina

Coordinateur assistanat au cabinet

Dr B. Brinkley
- Coordination du Cursus

- Liens avec les Facultés

- Coaching et «projets individuels»

- Lobby politique au niveau national

- Liens avec les associations cantonales

- Recherche de places de stage

- Réseau professionnel

- Lobby politique dans les cantons

\section{CURSUS ROMAND DE MÉDECINE DE FAMILLE}

Son site internet est complètement réactualisé à l'adresse www.devenirmedecindefamille.ch ou www.crmf.ch

\section{Naissance du CRMF, objectifs, structure et financement du projet}

Le 19 mai 2005, les médecins de famille romands sont interpellés par la conférence de presse de la CDS où la pénurie de médecins de famille qui menace la Suisse est rendue publique pour la première fois. En 2007, suite à diverses réflexions, se concrétise l'idée de reprendre les acquis et la structure du cursus de formation des généralistes mis au point à la Policlinique Médicale Universitaire de Lausanne dans le but de lui donner une dimension romande. C'est la naissance du Cursus Romand de Médecine Générale, aujourd'hui Cursus Romand de Médecine de famille.

Ce dernier poursuit trois objectifs:

- Encourager les étudiants et les collègues en formation à choisir la médecine de famille en promouvant l'attractivité de la médecine ambulatoire et en cabinet

- Favoriser la décentralisation et l'installation en régions périphériques pour une répartition uniforme des médecins entre zones urbaines et périphériques romandes

- Assurer le développement et l'intégration de l'assistanat au cabinet médical

Son organisation mise sur la régionalisation et la décentralisation.

Concrètement, le CRMF entre en contact avec les étudiants des facultés et les médecins assistants candidats à une formation en médecine de famille. Ces derniers reçoivent des conseils et un suivi sous forme d'entretiens individuels et personnalisés avec des médecins de famille coordinateurs. Le but est de construire leur programme de formation et choisir leurs places de formation postgraduée en cohérence avec le règlement, leurs intérêts, leur projet professionnel et leur réalité privée. Ces coordinateurs sont rattachés soit aux Facultés de Médecine de Lausanne 


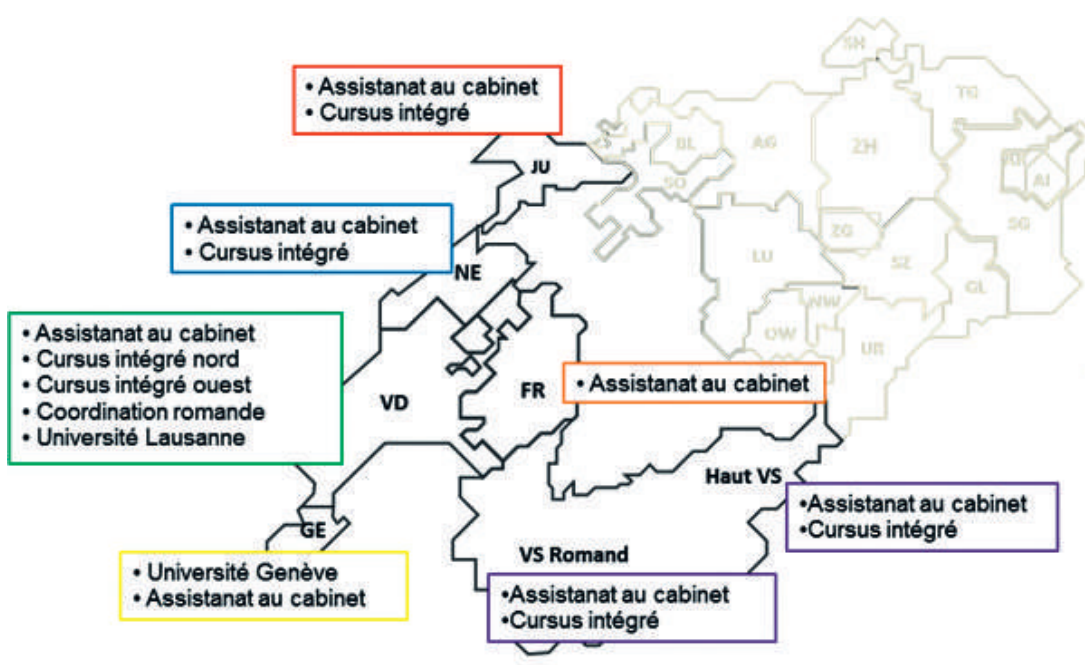

Figure 1

Le cœur de l'organisation du CRMF: un réseau régional de cursus intégrés et de coordinateurs, relié en son centre auprès des facultés de Médecine de Lausanne et Genève.

1 Martin S. Forum devenirgeneraliste.ch 2012 une vitrine pour la relève sur la formation, la pratique et la carrière en médecine de famille. PrimaryCare. 2012;12(12):220

\section{CLASS pour CRMF}

\section{CLASS}

- Pour projet d'assistanat au cabinet médical

48000

- Future évaluation du projet

10000

Société Suisse de Médecine Générale

5000

Société Suisse de Médecine Interne

7500

Association des médecins assistants et chefs de clinique vaudois

MF-Vaud

5000

Total

188000
Pour son financement, le CRMF compte sur le soutien des Départements de la Santé des cantons de Suisse romande par l'intermédiaire de la Conférence Latine des Directeurs de l'Action Sociale et de la Santé, de la SSMI, de la SSMG, de l'ASMAV et de MF Vaud (tabl. 2).

Les associations cantonales de médecins de famille des cantons romands et les Policliniques Médicales Universitaires participent à l'effort en offrant soutien logistique et travail en nature.

Les cursus intégrés bénéficient de budgets spécifiques attribués localement.

\section{La régionalisation: pierre angulaire de l'action sur le terrain}

Outre le suivi et les conseils dont profitent ou ont profité 250 médecins ayant choisi une formation en médecine de famille depuis 2007 , les médecins coordinateurs du CRMF se sont attelés à soutenir la création dans tous les cantons romands de cursus intégrés. Ces cursus sont centrés sur une collaboration avec les hôpitaux régionaux et cantonaux et sont gérés par des médecins de famille nommés médecins cadres à temps partiel de ces hôpitaux. Ils intègrent un programme local ou cantonal d'assistanat au cabinet. Les médecins assistants effectuent, sous un seul contrat, un tournus personnalisé de 2 à 3 ans dans plusieurs disciplines et bénéficient du programme local ou cantonal d'assistanat au cabinet médical. A ce jour, c'est ce réseau régional de cursus intégrés et de coordinateurs, relié en son centre auprès des facultés de Médecine de Lausanne et Genève, qui constitue le cœur de l'organisation du CRMF sur le terrain (fig. 1).

\section{Un pari pour l'avenir}

Toute cette organisation s'appuie à ce jour sur le pari suivant: développer des forces au niveau régional (Romandie) stimule les initiatives locales et encourage les médecins assistants à choisir une formation postgraduée en médecine de famille. Les formateurs sont encouragés à soigner la pertinence de leur offre de formation pour les médecins de famille dans les différents services hospitaliers et ambulatoires. Dans la foulée, les médecins installés ouvrent leurs cabinets et sont encouragés à développer l'assistanat au cabinet. Dans ces conditions, les jeunes collègues sont encouragés à choisir cette formation postgraduée et ceci stimule rétroactivement tout le système. Ce pari implique un investissement dont le résultat est à ce jour incertain, même si les premiers indicateurs incitent à l'optimisme. Le Cursus Romand de Médecine de famille se donne pour mission à l'avenir d'évaluer l'impact de son action sur la formation postgraduée en médecine de famille et de créer de la connaissance dans ce domaine.

Si ce modèle de régionalisation trouvait un écho dans d'autres parties de Suisse, le CRMF pourrait alors s'engager à démontrer prochainement que son pari est un pari gagnant! 